\title{
Determining The Number of Connected Vertex Labeled Graphs of Order Seven without Loops by Observing The Patterns of Formula for Lower Order Graphs with Similar Property
}

\author{
Muslim Ansori ${ }^{1}$, Wamiliana ${ }^{1 *}$, Fadila Cahya Puri ${ }^{1}$ \\ ${ }^{1}$ Department of Mathematics, Faculty of Mathematics and Natural Science, Universitas Lampung, Bandar Lampung, 35145, Indonesia, \\ *Corresponding author: wamiliana.1963@fmipa.unila.ac.id
}

\begin{abstract}
Given $n$ vertices and $m$ edges, $m \geq 1$, and for every vertex is given a label, there are lots of graphs that can be obtained. The graphs obtained may be simple or not simple, connected or disconnected. A graph $G(V, E)$ is called simple if $G(V, E)$ not containing loops nor paralel edges. An edge which has the same end vertex is called a loop, and paralel edges are two or more edges which connect the same set of vertices. Let $N\left(G_{7, m, t}\right)$ as the number of connected vertex labeled graphs of order seven with $m$ vertices and $t$ ( $t$ is the number edges that connect different pair of vertices). The result shows that $N\left(G_{7, m, t}\right)=c_{t} C_{t-1}^{(m-1)}$, with $c_{6}=6727, c_{7}=$ $30160, c_{8}=30765, c_{9}=21000, c_{10}=28364, c_{11}=26880, c_{12}=26460, c_{13}=20790, c_{14}=10290, c_{15}=8022, c_{16}=2940, c_{17}=4417, c_{18}=2835$, $c_{19}=210, c_{20}=21, c_{21}=1$.
\end{abstract}

Keywords

Graph, Connected, Vertex, Labeled, Order, Loops

Received: 7 July 2021, Accepted: 4 October 2021

https://doi.org/10.26554/sti.2021.6.4.328-336

\section{INTRODUCTION}

Graph theory emerged as a new field in mathematics in 1736 after Leonhard Euler gave solution to the Konigsberg problem, graph theory was used widely in many real-life applications, especially as problems representation. A Graph $\mathrm{G}(\mathrm{V}, \mathrm{E})$ is a structure which consists of a set $\mathrm{V}=\left\{v_{1}, v_{2}, \ldots, v_{n}\right\}$ of vertices, where $\mathrm{V} \neq \varnothing$, and a set of edges $\mathrm{E}=\mathrm{e}_{i j} \mid \mathrm{i}, \mathrm{j} \in \mathrm{V}$ which connect the vertices of $\mathrm{V}$. Usually the vertices are used to represent cities, depots, train stations, airports, etc., while edges are usually used to represent roads, train tracks, flight paths, etc. A number $\mathrm{c}_{i j} \geq 0$ can be assigned to the edge $\mathrm{e}_{i j}$ as a nonformal information which can represent the distance, time, cost, flow, etc. Because the flexibility of how to draw a graph, where there is no restriction in drawing an edge (can be a straight line, a curve, or other line), graph becomes an interesting structure to cope with, especially to represent the problem for easily visualization. Some of graph terminologies that commonly used in application is the concept of tree, where tree is a connected graph without cycle.

Some applications that use graph theoretical concept as problems representation include applications in biology, chemistry, engineering, computer science, economics, agriculture, and others. For example, in biology, a leaf labeled tree was used to represent the evolutionary history of a set of taxa which is called as phylogenetic tree (Huson and Bryant, 2006; Brandes and Cornelsen, 2009), and Mathur and Adlakha (2016) used combined tree to represent DNA, in chemistry/pharmaceutical, Gramatica et al. (2014) used graph concept to describe or represent the possible modes of action for any given pharmacological compound; in engineering and computer science, Hsu and Lin (2009) exposed a lot of graph theoretical concepts including Hamiltonian circuits with relation in network design, $\mathrm{Al}$ Etaiwi (2014) in order to generate a complex cipher text used the concepts minimum spanning tree, complete graph and cycle graph, Priyadarsini (2015) investigate the use of graph theory concept, extremal and expander graphs in designing some ciphers, while Ni et al. (2021) use bipartite and corona graphs to create ciphers; in economics, Álvarez and Ehnts (2015) used directed graph to represent the dynamic closures of the accounting structure; in agriculture, Kawakura and Shibasaki (2018) used graph theory concepts to group agricultural workers engaging in manual tasks, Kannimuthu et al. (2020) use graph coloring to optimize farmer's objective, and many more.

In 1857 Cayley enumerated the isomer of $\mathrm{C}_{n} \mathrm{H}_{2 n+2}$ using the concept of tree (Cayley, 1874), and followed by Slomenski (1964) who used graph theory to calculate additive structural 
properties of hydrocarbon. Bona (2007) discussed how to enumerate trees and forest. If we are given $n$ vertices and m edges, then lots of graphs can be obtained using that information. The graph obtained may be simple graph which does not contain loop nor parallel edges, or maybe not simple. Moreover, the graph obtained also may be connected or disconnected. For connected vertex labeled graph, the number of graph of order five with maximum number of paralel edges is five without loops was investigated by Wamiliana et al. (2019), and the number of graph of order six without paralel edges with ten loops maximum also investigated by Wamiliana et al. (2020). Puri et al. (2021) investigated the number of graphs of order six with maximum thirty edges without loops. For disconnected vertex labeled graph, Wamiliana et al. (2016) investigated the number of graph of order five without paralel edges, Amanto et al. (2017) gave the formula for graph of order maximal four, Putri et al. (2021) observed and gave formula for the number graphs of order six without loops and may contain maximum twenty parallel edges, while Pertiwi et al. (2021) proposed the formula for counting the number graph of order six without loops, especially when the graph obtained only contains maximum seven loops and the number of non loop edges is even. In this study, by observing the patterns of the formula of the number of connected vertex labeled graphs of order five and order six containing no loops, the formula of graphs of order seven with similar property will be discussed.

We organized this paper as follows: Section I is Introduction that describes about what is graph, some applications of graphs, and some researches related with this study. In Section II Observation and Investigation will be discussed while Result and Discussion is provided in Section III, and Conclusion in given in Section IV.

\section{OBSERVATION AND INVESTIGATION}

Given a graph $\mathrm{G}(\mathrm{V}, \mathrm{E})$ where $\mathrm{n}=|\mathrm{V}|=7$ and $\mathrm{G}$ is connected. Because $G$ is connected, then the number of edges $m=|E| \geq$ 6. Every vertex in $G$ is labeled, therefore graphs $G_{1}$ and $G_{2}$ in Figure 1 are two different graphs even though both graphs look similar.

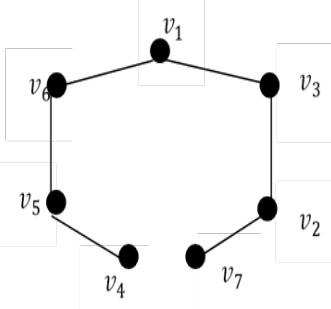

$G_{1}$

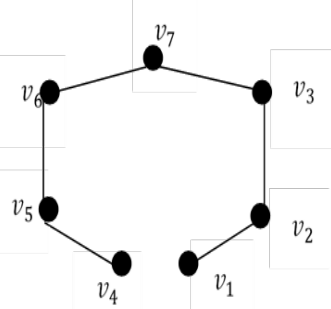

$G_{2}$

Figure 1. Two Different Graphs that Look The Same but Different because of The Vertex Labeling
Denote $\mathrm{N}\left(\mathrm{G}_{n, m, t}\right)$ as the number of connected vertex labeled graphs containing no loops of order $n, m$ edges and $t$, where $t$ is the number of edges that connect different pairs of vertices in $\mathrm{G}$. Edges that connect the same pair of vertices is counted as one. Moreover, isomorphics graphs are counted as one graph.

The result on Table 1 for $\mathrm{n}=5$ and are obtained from Wamiliana et al. (2019), and for $\mathrm{n}=6$ from and Puri et al. (2021). From Table 1 we know that for $\mathrm{n}=5$, the maximum number of $\mathrm{t}$ is 10 , and for $\mathrm{n}=6$ is 15 , and since the graph is connected, then $m \geq 4$ for $n=5$, and $m \geq 5$ for $n=6$. By observing Table 1 we found that there are patterns between those two order graphs. Notice that, for every $t$, the formula only differ on the coefficients. Let ct is contant with $t=6,7, \ldots, 21$. By using the patterns on Table 1, we predict that the formula for order seven as $c_{t} C_{t-1}^{(m-1)}$. Note that for order 7 , maximum $\mathrm{t}$ is 21 .

\section{RESULTS AND DISGUSSION}

Given $n=7, t$ and $m$, the number of graphs of order seven, connected, and vertex labelled are obtained by: pattern construction, grouping the patterns in term of $\mathrm{m}$ and $\mathrm{t}$, and then calculate the graphs. Starting with $\mathrm{t}=6$, we construct for $\mathrm{m} \geq 6$. The process continue with $\mathrm{t}=7$ until $\mathrm{t}=21$ (maximum possible $\mathrm{t}$ for $n=7$ ). Table 2 shows some possible patterns for $t=n-1$.

Note that we do not put all possible patterns here due to space limitation, for example, for $\mathrm{t}=\mathrm{n}-1$ and $\mathrm{m}=\mathrm{n}$, the paralel edges maybe connect vertex $v_{1}$ and $v_{2}$ or $v_{4}$ and $v_{5}$, and so on, and for $\mathrm{t}=\mathrm{n}-1$ and $\mathrm{m}=\mathrm{n}+1$, that is possible the paralel edges only on one pair of vertices, for example, there are three edges that connect vertex $v_{1}$ and $v_{2}$, etc. The number of graphs obtained is given in Table 3 . By observing the number in every column, Table 3 can be rewrite as in Table 4 .

By grouping the graphs by $m$ and $t$, we notice that every column of Table 4 constitute patterns. Note that in the Table 3 and 4 we are not inputting all the numbers of graph obtained because $t$ is fixed in every column and adding more edges only adding more paralel edges on $\mathrm{t}$, and the pattern continues for the next $\mathrm{m}$, for example: for $t=6, m \geq 6$ we only input the number until $m=12$ and the pattern is $1,6,21,56,126,252$, 462 (if adding more $m$, the pattern becomes $1,6,21,56,126$, $252,462,792,1287,2002, \ldots)$.

The sequence of numbers that appear in the first column $(t=$ $6)$ is $1,6,21,56,126,252,462$ and that number is multiplied by 6727 . Therefore we can claim that the value of $c_{6}$ in Table 3 is 6727 .

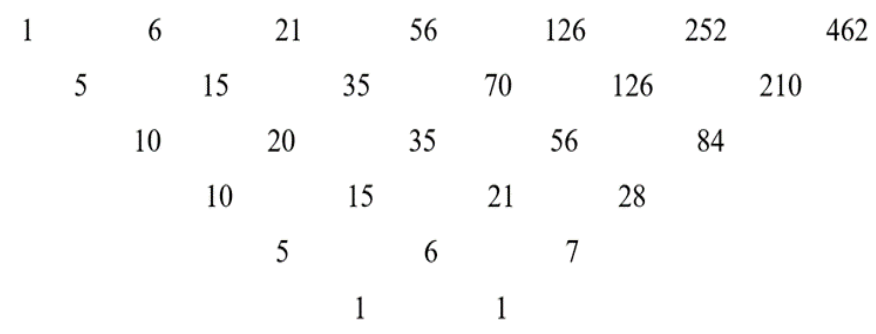

Page 329 of 336 
Table 1. The Formula of The Number of Connected Vertex Labeled Graph of Order N , N = 5, 6, M Edges And T, where T is The Number of Edges that Connect Different Pairs of Vertices in Graph, and Containing no Loops

\begin{tabular}{ccc}
\hline & \multicolumn{2}{c}{$\mathrm{n}$} \\
$\mathrm{t}$ & \multicolumn{1}{c}{5} & 6 \\
\hline 4 & $\mathrm{~N}\left(\mathrm{G}_{5, m, 4}\right)=125 \times C_{3}^{(m-1)}$ & \\
5 & $\mathrm{~N}\left(\mathrm{G}_{5, m, 5}\right)=222 \times C_{4}^{(m-1)}$ & $\mathrm{N}\left(\mathrm{G}_{6, m, 5}\right)=1296 \times C_{4}^{(m-1)}$ \\
6 & $\mathrm{~N}\left(\mathrm{G}_{5, m, 6}\right)=205 \times C_{5}^{(m-1)}$ & $\mathrm{N}\left(\mathrm{G}_{6, m, 6}\right)=1980 \times C_{5}^{(m-1)}$ \\
7 & $\mathrm{~N}\left(\mathrm{G}_{5, m, 7}\right)=110 \times C_{6}^{(m-1)}$ & $\mathrm{N}\left(\mathrm{G}_{6, m, 7}\right)=3330 \times C_{6}^{(m-1)}$ \\
8 & $\mathrm{~N}\left(\mathrm{G}_{5, m, 8}\right)=45 \times C_{7}^{(m-1)}$ & $\mathrm{N}\left(\mathrm{G}_{6, m, 8}\right)=4620 \times C_{7}^{(m-1)}$ \\
9 & $\mathrm{~N}\left(\mathrm{G}_{5, m, 9}\right)=10 \times C_{8}^{(m-1)}$ & $\mathrm{N}\left(\mathrm{G}_{6, m, 9}\right)=6660 \times C_{8}^{(m-1)}$ \\
10 & $\mathrm{~N}\left(\mathrm{G}_{5, m, 10}\right)=1 \times C_{9}^{(m-1)}$ & $\mathrm{N}\left(\mathrm{G}_{6, m, 10}\right)=2640 \times C_{9}^{(m-1)}$ \\
11 & & $\mathrm{~N}\left(\mathrm{G}_{6, m, 11}\right)=1155 \times C_{10}^{(m-1)}$ \\
12 & & $\mathrm{~N}\left(\mathrm{G}_{6, m, 12}\right)=420 \times C_{11}^{(m-1)}$ \\
13 & & $\mathrm{~N}\left(\mathrm{G}_{6, m, 13}\right)=150 \times C_{12}^{(m-1)}$ \\
14 & & $\mathrm{~N}\left(\mathrm{G}_{6, m, 14}\right)=15 \times C_{13}^{(m-1)}$ \\
15 & & $\mathrm{~N}\left(\mathrm{G}_{6, m, 13}\right)=1 \times C_{14}^{(m-1)}$ \\
\hline
\end{tabular}

Table 2. Some Possible Patterns for $\mathrm{t}=\mathrm{n}-1(\mathrm{n}=7)$

t=n-l,m=n-I

Table 3. Grouping The Number of Connected Vertex Labeled Graph of Order Seven without Loops by $m$ and $t$

\begin{tabular}{ccccccc}
\hline \multicolumn{6}{c}{ The Number of Connected Vertex Labeled Graphs of Order Seven without Loops } \\
$\mathrm{m}$ & 6 & 7 & 8 & $\mathrm{t}$ & 10 & 11 \\
\hline 6 & 6727 & & & & & \\
7 & 40362 & 30160 & & & & \\
8 & 141267 & 211120 & 30765 & & & \\
9 & 376712 & 844480 & 246120 & 21000 & & \\
10 & 847602 & 2533440 & 1107540 & 189000 & 28364 & \\
11 & 1695204 & 6333600 & 3691800 & 945000 & 283640 & 26880 \\
12 & 3107874 & 13933920 & 10152450 & 3465000 & 1560020 & 295680 \\
13 & & 27867840 & 24365880 & 10395000 & 6240080 & 1774080 \\
14 & & 51754560 & 52792740 & 27027000 & 20280260 & 7687680 \\
15 & & & 105585480 & 63063000 & 56784728 & 26906880 \\
16 & & & 197972775 & 135135000 & 141961820 & 80720640 \\
17 & & & 270270000 & 324484160 & 215255040 \\
18 & & & 510510000 & 689528840 & 522762240 \\
19 & & & & 1379057680 & 1176215040 \\
20 & & & & & 2620209592 & 2483120640 \\
21 & & & & & 4966241280 \\
22 & & & & & & 9481006080 \\
\hline
\end{tabular}




\begin{tabular}{|c|c|c|c|c|c|}
\hline \multirow[b]{2}{*}{$\mathrm{m}$} & \multicolumn{5}{|c|}{ The Number of Connected Vertex Labeled Graphs of Order Seven without Loops } \\
\hline & 12 & 13 & 14 & 15 & 16 \\
\hline 12 & 26460 & & & & \\
\hline 13 & 317520 & 20790 & & & \\
\hline 14 & 2063880 & 270270 & 10290 & & \\
\hline 15 & 9631440 & 1891890 & 144060 & 8022 & \\
\hline 16 & 36117900 & 9459450 & 1080450 & 120330 & 5460 \\
\hline 17 & 115577280 & 37837800 & 5762400 & 962640 & 87360 \\
\hline 18 & 327468960 & 128648520 & 24490200 & 5454960 & 742560 \\
\hline 19 & 842063040 & 385945560 & 88164720 & 24547320 & 4455360 \\
\hline 20 & 1999899720 & 1047566520 & 279188280 & 93279816 & 21162960 \\
\hline 21 & 4444221600 & 2618916300 & 797680800 & 310932720 & 84651840 \\
\hline 22 & 9332865360 & 6110804700 & 2093912100 & 932798160 & 296281440 \\
\hline 23 & 18665730720 & 13443770340 & 5118451800 & 2565194940 & 931170240 \\
\hline 24 & 35775983880 & 28109701620 & 11772439140 & 6555498180 & 2677114440 \\
\hline 25 & & 56219403240 & 25685321760 & 15733195632 & 7138971840 \\
\hline 26 & & 108114237000 & 53511087000 & 35757262800 & 17847429600 \\
\hline 27 & & & 107022174000 & 77474069400 & 42184833600 \\
\hline 28 & & & 206399907000 & 160907682600 & 94915875600 \\
\hline 29 & & & & 321815365200 & 204434193600 \\
\hline 30 & & & & 622176372720 & 423470829600 \\
\hline 31 & & & & & 846941659200 \\
\hline 32 & & & & & 1640949464700 \\
\hline
\end{tabular}

\begin{tabular}{|c|c|c|c|c|c|}
\hline \multirow[b]{2}{*}{$\mathrm{m}$} & \multicolumn{5}{|c|}{ The Number of Connected Vertex Labeled Graphs of Order Seven without Loops } \\
\hline & 17 & 18 & 19 & 20 & 21 \\
\hline 17 & 4417 & & & & \\
\hline 18 & 75089 & 2835 & & & \\
\hline 19 & 675801 & 51030 & 210 & & \\
\hline 20 & 4280073 & 484785 & 3990 & 21 & \\
\hline 21 & 21400365 & 3231900 & 39900 & 420 & 1 \\
\hline 22 & 89881533 & 16967475 & 279300 & 4410 & 21 \\
\hline 23 & 329565621 & 74656890 & 1536150 & 32340 & 231 \\
\hline 24 & 1082858469 & 286184745 & 7066290 & 185955 & 1771 \\
\hline 25 & 3248575407 & 981204840 & 28265160 & 892584 & 10626 \\
\hline 26 & 9023820575 & 3066265125 & 100947000 & 3719100 & 53130 \\
\hline 27 & 23461933495 & 8858099250 & 328077750 & 13813800 & 230230 \\
\hline 28 & 57588382215 & 23916867975 & 984233250 & 46621575 & 888030 \\
\hline 29 & 134372891835 & 60879300300 & 2755853100 & 145044900 & 3108105 \\
\hline 30 & 299754912555 & 147124975725 & 7265430900 & 420630210 & 10015005 \\
\hline 31 & 642331955475 & 339519174750 & 18163577250 & 1147173300 & 30045015 \\
\hline 32 & 1327486041315 & 751792458375 & 43313145750 & 2963531025 & 84672315 \\
\hline 33 & 2654972082630 & 1603823911200 & 99001476000 & 7294845600 & 225792840 \\
\hline 34 & 5153769336870 & 3307886816850 & 217803247200 & 17194993200 & 573166440 \\
\hline 35 & & 6615773633700 & 462831900300 & 38975317920 & 1391975640 \\
\hline 36 & & 12864004287750 & 952889206500 & 85258507950 & 3247943160 \\
\hline 37 & & & 1905778413000 & 180547428600 & 7307872110 \\
\hline 38 & & & 3711252699000 & 371125269900 & 15905368710 \\
\hline 39 & & & & 742250539800 & 33578000610 \\
\hline 40 & & & & 1447388552610 & 68923264410 \\
\hline 41 & & & & & 137846528820 \\
\hline 42 & & & & & 269128937220 \\
\hline
\end{tabular}


Table 4. Another form of Table 3

\begin{tabular}{|c|c|c|c|c|c|c|}
\hline \multirow[b]{3}{*}{$\mathrm{m}$} & \multicolumn{6}{|c|}{ The Number of Connected Vertex Labeled Graphs of Order Seven without Loops } \\
\hline & & & & $\mathrm{t}$ & & \\
\hline & 6 & 7 & 8 & 9 & 10 & 11 \\
\hline 6 & $1 \times 6727$ & & & & & \\
\hline 7 & $6 \times 6727$ & $1 \times 30160$ & & & & \\
\hline 8 & $21 \times 6727$ & $7 \times 30160$ & $1 \times 30765$ & & & \\
\hline 9 & $56 \times 6727$ & $28 \times 30160$ & $8 \times 30765$ & $1 \times 21000$ & & \\
\hline 10 & $126 \times 6727$ & $84 \times 30160$ & $36 \times 30765$ & $9 \times 21000$ & $1 \times 28364$ & \\
\hline 11 & $252 \times 6727$ & $210 \times 30160$ & $120 \times 30765$ & $45 \times 21000$ & $10 \times 28364$ & $1 \times 26880$ \\
\hline 12 & $462 \times 6727$ & $462 \times 30160$ & $330 \times 30765$ & $165 \times 21000$ & $55 \times 28364$ & $11 \times 26880$ \\
\hline 13 & & $924 \times 30160$ & $792 \times 30765$ & $495 \times 21000$ & $220 \times 28364$ & $66 \times 26880$ \\
\hline 14 & & $1716 \times 30160$ & $1716 \times 30765$ & $1287 \times 21000$ & $715 \times 28364$ & $286 \times 26880$ \\
\hline 15 & & & $3432 \times 30765$ & $3003 \times 21000$ & $2002 \times 28364$ & $1001 \times 26880$ \\
\hline 16 & & & $6435 \times 30765$ & $6435 \times 21000$ & $5005 \times 28364$ & $3003 \times 26880$ \\
\hline 17 & & & & $12870 \times 21000$ & $11440 \times 28364$ & $8008 \times 26880$ \\
\hline 18 & & & & $24310 \times 21000$ & $24310 \times 28364$ & $19448 \times 26880$ \\
\hline 19 & & & & & $48620 \times 28364$ & $43758 \times 26880$ \\
\hline 20 & & & & & $92378 \times 28364$ & $92378 \times 26880$ \\
\hline 21 & & & & & & $184756 \times 26880$ \\
\hline 22 & & & & & & $352716 \times 26880$ \\
\hline
\end{tabular}

The Number of Connected Vertex Labeled Graphs of Order Seven without Loops

\begin{tabular}{|c|c|c|c|c|c|}
\hline $\mathrm{m}$ & 12 & 13 & $\begin{array}{r}\mathrm{t} \\
14\end{array}$ & 15 & 16 \\
\hline 12 & $1 \times 26460$ & & & & \\
\hline 13 & $12 \times 26460$ & $1 \times 20790$ & & & \\
\hline 14 & $78 \times 26460$ & $13 \times 20790$ & $1 \times 10290$ & & \\
\hline 15 & $364 \times 26460$ & $91 \times 20790$ & $14 \times 10290$ & $1 \times 8022$ & \\
\hline 16 & $1365 \times 26460$ & $455 \times 20790$ & $105 \times 10290$ & $15 \times 8022$ & $1 \times 2940$ \\
\hline 17 & $4368 \times 26460$ & $1820 \times 20790$ & $560 \times 10290$ & $120 \times 8022$ & $16 \times 2940$ \\
\hline 18 & $12376 \times 26460$ & $6188 \times 20790$ & $2380 \times 10290$ & $680 \times 8022$ & $136 \times 2940$ \\
\hline 19 & $31824 \times 26460$ & $18564 \times 20790$ & $8568 \times 10290$ & $3060 \times 8022$ & $816 \times 2940$ \\
\hline 20 & $75582 \times 26460$ & $50388 \times 20790$ & $27132 \times 10290$ & $11628 \times 8022$ & $3876 \times 2940$ \\
\hline 21 & $167960 \times 26460$ & $125970 \times 20790$ & $77520 \times 10290$ & $38760 \times 8022$ & $15504 \times 2940$ \\
\hline 22 & $352716 \times 26460$ & $293930 \times 20790$ & $203490 \times 10290$ & $116280 \times 8022$ & $54264 \times 2940$ \\
\hline 23 & $705432 \times 26460$ & $646646 \times 20790$ & $497420 \times 10290$ & $319770 \times 8022$ & $170544 \times 2940$ \\
\hline 24 & $1352078 \times 26460$ & $1352078 \times 20790$ & $1144066 \times 10290$ & $817190 \times 8022$ & $490314 \times 2940$ \\
\hline 25 & & $2704156 \times 20790$ & $2496144 \times 10290$ & $1961256 \times 8022$ & $1307504 \times 2940$ \\
\hline 26 & & $5200300 \times 20790$ & $5200300 \times 10290$ & $4457400 \times 8022$ & $3268760 \times 2940$ \\
\hline 27 & & & $10400600 \times 10290$ & $9657700 \times 8022$ & $7726160 \times 2940$ \\
\hline 28 & & & $20058300 \times 10290$ & $20058300 \times 8022$ & $17383860 \times 2940$ \\
\hline 29 & & & & $40116600 \times 8022$ & $37442160 \times 2940$ \\
\hline 30 & & & & $77558760 \times 8022$ & $77558760 \times 2940$ \\
\hline 31 & & & & & $155117520 \times 2940$ \\
\hline 32 & & & & & $300540195 \times 2940$ \\
\hline
\end{tabular}

Result 1: Given $\mathrm{n}=7, m \geq 6, t=6$, the number of connected graphs of order seven containing no loops is $\mathrm{N}\left(\mathrm{G}_{7, m, 6}\right)=6727$ $\times C_{5}^{(m-1)}$.

Proof:
Look at the sequence of numbers above.

It can be seen that from the sequence above that the fixed difference occur on the fifth level. Therefore the polynomial that can represent this sequence is polynomial of order five: 


\begin{tabular}{|c|c|c|c|c|c|}
\hline \multirow[b]{3}{*}{$\mathrm{m}$} & \multicolumn{5}{|c|}{ The Number of Connected Vertex Labeled Graphs of Order Seven } \\
\hline & & & $\mathrm{t}$ & & \\
\hline & 17 & 18 & 19 & 20 & 21 \\
\hline 17 & $1 \times 4417$ & & & & \\
\hline 18 & $17 \times 4417$ & $1 \times 2835$ & & & \\
\hline 19 & $153 \times 4417$ & $18 \times 2835$ & $1 \times 210$ & & \\
\hline 20 & $969 \times 4417$ & $171 \times 2835$ & $19 \times 210$ & $1 \times 21$ & \\
\hline 21 & $4845 \times 4417$ & $1140 \times 2835$ & $190 \times 210$ & $20 \times 21$ & $1 \times 1$ \\
\hline 22 & $20349 \times 4417$ & $5985 \times 2835$ & $1330 \times 210$ & $210 \times 21$ & $21 \times 1$ \\
\hline 23 & $74613 \times 4417$ & $26334 \times 2835$ & $7315 \times 210$ & $1540 \times 21$ & $231 \times 1$ \\
\hline 24 & $245157 \times 4417$ & $100947 \times 2835$ & $33649 \times 210$ & $8855 \times 21$ & $1771 \times 1$ \\
\hline 25 & $735471 \times 4417$ & $346104 \times 2835$ & $134596 \times 210$ & $42504 \times 21$ & $10626 \times 1$ \\
\hline 26 & $2042975 \times 4417$ & $1081575 \times 2835$ & $480700 \times 210$ & $177100 \times 21$ & $53130 \times 1$ \\
\hline 27 & $5311735 \times 4417$ & $3124550 \times 2835$ & $1562275 \times 210$ & $657800 \times 21$ & $230230 \times 1$ \\
\hline 28 & $13037895 \times 4417$ & $8436285 \times 2835$ & $4686825 \times 210$ & $2220075 \times 21$ & $888030 \times 1$ \\
\hline 29 & $30421755 \times 4417$ & $21474180 \times 2835$ & $13123110 \times 210$ & $6906900 \times 21$ & $3108105 \times 1$ \\
\hline 30 & $67863915 \times 4417$ & $51895935 \times 2835$ & $34597290 \times 210$ & $20030010 \times 21$ & $10015005 \times 1$ \\
\hline 31 & $145422675 \times 4417$ & $119759850 \times 2835$ & $86493225 \times 210$ & $54627300 \times 21$ & $30045015 \times 1$ \\
\hline 32 & $300540195 \times 4417$ & $265182525 \times 2835$ & $206253075 \times 210$ & $141120525 \times 21$ & $84672315 \times 1$ \\
\hline 33 & $601080390 \times 4417$ & $565722720 \times 2835$ & $471435600 \times 210$ & $347373600 \times 21$ & $225792840 \times 1$ \\
\hline 34 & $1166803110 \times 4417$ & $1166803110 \times 2835$ & $1037158320 \times 210$ & $818809200 \times 21$ & $573166440 \times 1$ \\
\hline 35 & & $2333606220 \times 2835$ & $2203961430 \times 210$ & $1855967520 \times 21$ & $1391975640 \times 1$ \\
\hline 36 & & $4537567650 \times 2835$ & $4537567650 \times 210$ & $4059928950 \times 21$ & $3247943160 \times 1$ \\
\hline 37 & & & $9075135300 \times 210$ & $8597496600 \times 21$ & $7307872110 \times 1$ \\
\hline 38 & & & $17672631900 \times 210$ & $17672631900 \times 21$ & $15905368710 \times 1$ \\
\hline 39 & & & & $35345263800 \times 21$ & $33578000610 \times 1$ \\
\hline 40 & & & & $68923264410 \times 21$ & $68923264410 \times 1$ \\
\hline 41 & & & & & $137846528820 \times 1$ \\
\hline 42 & & & & & $269128937220 \times 1$ \\
\hline
\end{tabular}

$P_{5}(m)=a_{5} m^{5}+a_{4} m^{4}+a_{3} m^{3}+a_{2} m^{2}+a_{1} m+a_{0}$

Substitute $m=6,7,8,9,10,11$ to the equation we get the following:

$$
\begin{gathered}
6727=7776 a_{5}+1296 a_{4}+216 a_{3}+36 a_{2}+6 a_{1}+a_{0} \\
40362=16807 a_{5}+2401 a_{4}+343 a_{3}+49 a_{2}+7 a_{1}+a_{0} \quad(2) \\
141267=32768 a_{5}+4096 a_{4}+512 a_{3}+64 a_{2}+8 a_{1}+a_{0} \quad(3) \\
376712=59049 a_{5}+6561 a_{4}+729 a_{3}+81 a_{2}+9 a_{1}+a_{0} \\
847602=100000 a_{5}+10000 a_{4}+1000 a_{3}+100 a_{2}+10 a_{1}+a_{0}
\end{gathered}
$$

$1695204=161051 a_{5}+14641 a_{4}+1331 a_{3}+121 a_{2}+$ $11 a_{1}+a_{0}$
Solving this system of linear equations we get $a_{5}=\frac{6727}{120}, a_{4}=$ $-\frac{100905}{120}, a_{3}=\frac{571795}{120}, a_{2}=-\frac{1513575}{120}, a_{1}=\frac{1843198}{120}$ and $a_{0}=$ $-\frac{807239}{120}$

$$
\begin{aligned}
P_{5}(m) & =a_{5} m^{5}+a_{4} m^{4}+a_{3} m^{3}+a_{2} m^{2}+a_{1} m+a_{0} \\
& =\frac{6727}{120} m^{5}-\frac{100905}{120} m^{4}+\frac{571795}{120} m^{3}-\frac{1513575}{120} m^{2} \\
& +\frac{1843198}{120} m-\frac{807239}{120} \\
& =\frac{6727}{120}\left(m^{5}-15 m^{4}+85 m^{3}-225 m^{2}+274 m-120\right) \\
& =\frac{6727}{120}(m-1)(m-2)(m-3)(m-4)(m-5) \\
& =6727 \times \frac{(m-1)(m-2)(m-3)(m-4)(m-5)}{(5 \times 4 \times 3 \times 2 \times 1)} \\
& =6727 \times C_{5}^{(m-1)}
\end{aligned}
$$

For $\mathrm{t}=7$, we can see from Table 4 that the sequence of numbers is $1,7,28,84,210,462,924,1716$. 


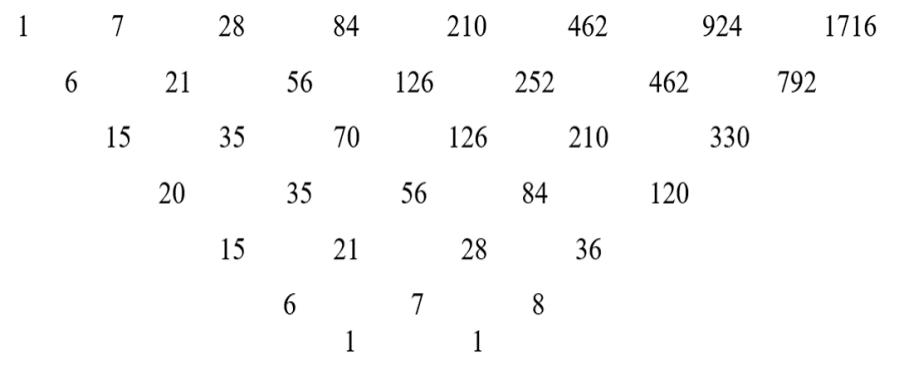

Result 2: Given $\mathrm{n}=7, m \geq 6, t=7$, the number of connected graphs of order seven containing no loops is $\mathrm{N}\left(\mathrm{G}_{7, m, 7}\right)=$ $30160 \times C_{6}^{(m-1)}$.

Proof:

Look at the sequence of numbers above.

It can be seen that from the sequence above that the fixed difference occur on the sixth level. Therefore the polynomial that can represent this sequence is polynomial of order six: $P_{6}(m)=a_{6} m^{6}+a_{5} m^{5}+a_{4} m^{4}+a_{3} m^{3}+a_{2} m^{2}+a_{1} m+a_{0}$ Substitute $m=7,8,9,10,11,12,13$ to the equation we get the following:

$30160=117649 a_{6}+16807 a_{5}+2401 a_{4}+343 a_{3}+49 a_{2}+7 a_{1}+a_{0}$

$211120=262144 a_{6}+32768 a_{5}+4096 a_{4}+512 a_{3}+64 a_{2}+8 a_{1}+a_{0}$

$844480=531441 a_{6}+59049 a_{5}+6561 a_{4}+729 a_{3}+81 a_{2}+9 a_{1}+a_{0}$

$2533440=1000000 a_{6}+100000 a_{5}+10000 a_{4}+1000 a_{3}+$ $100 a_{2}+10 a_{1}+a_{0}$

$6333600=1771561 a_{6}+161051 a_{5}+14641 a_{4}+1331 a_{3}+$ $121 a_{2}+11 a_{1}+a_{0}$

$13933920=2985984 a_{6}+248832 a_{5}+20736 a_{4}+1728 a_{3}$ $+144 a_{2}+12 a_{1}+a_{0}$

$$
\begin{aligned}
& 27867840=4826809 a_{6}+371293 a_{5}+28561 a_{4}+2197 a_{3} \\
& +169 a_{2}+13 a_{1}+a_{0}
\end{aligned}
$$

Solving this system of linear equations we get $a_{6}=\frac{30160}{720}, a_{5}=$ $-\frac{633360}{720}, a_{4}=\frac{5278000}{720}, a_{3}=-\frac{22167600}{720}, a_{2}=\frac{48979840}{720}, a_{1}=$ $-\frac{53202240}{720}$ and $a_{0}=\frac{21715200}{720}$

Therefore

$$
\begin{aligned}
P_{6}(m) & =a_{6} m^{6}+a_{5} m^{5}+a_{4} m^{4}+a_{3} m^{3}+a_{2} m^{2}+a_{1} m+a_{0} \\
& =\frac{30160}{720} m^{6}-\frac{633360 / 720}{720} m^{5}+\frac{5278000}{720} m^{4} \\
& -\frac{22167600}{720} m^{3}+\frac{48979840}{720} m^{2}-\frac{53202240}{720} m \\
& +\frac{21715200}{720} \\
& =\frac{30160}{720}\left(m^{6}-21 m^{5}+175 m^{4}-735 m^{3}+1624 m^{2}\right. \\
& -1764 m+720) \\
& =\frac{30160}{720}(m-1)(m-2)(m-3)(m-4)(m-5)(m-6) \\
& =30160 \times \frac{(m-1)(m-2)(m-3)(m-4)(m-5)(m-6)}{(6 \times 5 \times 4 \times 3 \times 2 \times 1)} \\
& =30160 \times C_{6}^{(m-1)} \quad
\end{aligned}
$$

Doing with similar manner we get the following results:

For $n=7, m \geq 8, t=8, N\left(G_{7, m, 8}\right)=30765 \times C_{7}^{(m-1)}$

For $n=7, m \geq 9, t=9, N\left(G_{7, m, 9}\right)=21000 \times C_{8}^{(m-1)}$

For $n=7, m \geq 10, t=10, N\left(G_{7, m, 10}\right)=28364 \times C_{9}^{(m-1)}$

For $n=7, m \geq 11, t=11, N\left(G_{7, m, 11}\right)=26880 \times C_{10}^{(m-1)}$

For $n=7, m \geq 12, t=12, N\left(G_{7, m, 12}\right)=26460 \times C_{11}^{(m-1)}$

For $n=7, m \geq 13, t=13, N\left(G_{7, m, 13}\right)=20790 \times C_{12}^{(m-1)}$

For $n=7, m \geq 14, t=14, N\left(G_{7, m, 14}\right)=10290 \times C_{13}^{(m-1)}$

For $n=7, m \geq 15, t=15, N\left(G_{7, m, 15}\right)=8022 \times C_{14}^{(m-1)}$

For $n=7, m \geq 16, t=16, N\left(G_{7, m, 16}\right)=2940 \times C_{15-1)}^{(m-1)}$

For $n=7, m \geq 17, t=17, N\left(G_{7, m, 17}\right)=4417 \times C_{16}^{(m-1)}$

For $n=7, m \geq 18, t=18, N\left(G_{7, m, 18}\right)=2835 \times C_{17}^{(m-1)}$

For $n=7, m \geq 19, t=19, N\left(G_{7, m, 19}\right)=210 \times C_{18}^{(m-1)}$

For $n=7, m \geq 20, t=20, N\left(G_{7, m, 20}\right)=21 \times C_{19}^{(m-1)}$

For $n=7, m \geq 21, t=21, N\left(G_{7, m, 21}\right)=1 \times C_{20}^{(m-1)}$

Base on these result, we get Table 5. From Table 5 it can be seen that for every $\mathrm{t}$, the formula consist of $C_{t-1}^{(m-1)}$, and the difference is on $c_{t}$. 
Table 5. Comparison for The Number of Connected Vertex Labeled Graphs of Order Five, Six, and Seven Containing no Loops

\begin{tabular}{cccc}
\hline & & $\mathrm{n}$ & \\
$\mathrm{t}$ & 5 & 6 & 7 \\
\hline 4 & $\mathrm{~N}\left(G_{5, m, 4}\right)=125 \times C_{3}^{(m-1)}$ & & \\
5 & $\mathrm{~N}\left(G_{5, m, 5}\right)=222 \times C_{4}^{(m-1)}$ & $\mathrm{N}\left(G_{6, m, 5}\right)=1296 \times C_{4}^{(m-1)}$ & \\
6 & $\mathrm{~N}\left(G_{5, m, 6}\right)=205 \times C_{5}^{(m-1)}$ & $\mathrm{N}\left(G_{6, m, 6}\right)=1980 \times C_{5}^{(m-1)}$ & $\mathrm{N}\left(G_{7, m, 6}\right)=6727 \times C_{5}^{(m-1)}$ \\
7 & $\mathrm{~N}\left(G_{5, m, 7}\right)=110 \times C_{6}^{(m-1)}$ & $\mathrm{N}\left(G_{6, m, 7}\right)=3330 \times C_{6}^{(m-1)}$ & $\mathrm{N}\left(G_{7, m, 7}\right)=30160 \times C_{6}^{(m-1)}$ \\
8 & $\mathrm{~N}\left(G_{5, m, 8}\right)=45 \times C_{7}^{(m-1)}$ & $\mathrm{N}\left(G_{6, m, 8}\right)=4620 \times C_{7}^{(m-1)}$ & $\mathrm{N}\left(G_{7, m, 8}\right)=30765 \times C_{7}^{(m-1)}$ \\
9 & $\mathrm{~N}\left(G_{5, m, 9}\right)=10 \times C_{8}^{(m-1)}$ & $\mathrm{N}\left(G_{6, m, 9}\right)=6660 \times C_{8}^{(m-1)}$ & $\mathrm{N}\left(G_{7, m, 9}\right)=21000 \times C_{8}^{(m-1)}$ \\
10 & $\mathrm{~N}\left(G_{5, m, 10}\right)=1 \times C_{9}^{(m-1)}$ & $\mathrm{N}\left(G_{6, m, 10}\right)=2640 \times C_{9}^{(m-1)}$ & $\mathrm{N}\left(G_{7, m, 10}\right)=28634 \times C_{9}^{(m-1)}$ \\
11 & & $\mathrm{~N}\left(G_{6, m, 11}\right)=1155 \times C_{10}^{(m-1)}$ & $\mathrm{N}\left(G_{7, m, 11}\right)=26880 \times C_{10}^{(m-1)}$ \\
12 & & $\mathrm{~N}\left(G_{6, m, 12}\right)=420 \times C_{11}^{(m-1)}$ & $\mathrm{N}\left(G_{7, m, 12}\right)=26460 \times C_{11}^{(m-1)}$ \\
13 & & $\mathrm{~N}\left(G_{6, m, 13}\right)=150 \times C_{12}^{(m-1)}$ & $\mathrm{N}\left(G_{7, m, 13}\right)=20790 \times C_{12}^{(m-1)}$ \\
14 & $\mathrm{~N}\left(G_{6, m, 14}\right)=15 \times C_{13}^{(m-1)}$ & $\mathrm{N}\left(G_{7, m, 14}\right)=10290 \times C_{13}^{(m-1)}$ \\
15 & & $\mathrm{~N}\left(G_{6, m, 13}\right)=1 \times C_{14}^{(m-1)}$ & $\mathrm{N}\left(G_{7, m, 15}\right)=8022 \times C_{14}^{(m-1)}$ \\
16 & & & $\mathrm{~N}\left(G_{7, m, 16}\right)=2940 \times C_{15}^{(m-1)}$ \\
17 & & $\mathrm{~N}\left(G_{7, m, 17}\right)=4417 \times C_{16}^{(m-1)}$ \\
18 & & & $\mathrm{~N}\left(G_{7, m, 18}\right)=2835 \times C_{17}^{(m-1)}$ \\
19 & & & $\mathrm{~N}\left(G_{7, m, 19}\right)=210 \times C_{18}^{(m-1)}$ \\
20 & & & $\mathrm{~N}\left(G_{7, m, 20}\right)=21 \times C_{19}^{(m-1)}$ \\
21 & & & $\mathrm{~N}\left(G_{7, m, 21}\right)=1 \times C_{20}^{(m-1)}$ \\
\hline
\end{tabular}

\section{CONCLUSIONS}

From the discussion above we can conclude that the formula to count the number of connected vertex labeled graph of order seven has a similar pattern with the lower order graph with the similar property. The difference of the formulas is on the coeficient for every t. The result shows that the number of connected vertex labeled graphs of order seven containing no loops is $N\left(G_{7, m, t}\right)=c_{t} C_{t-1}^{(m-1)}$, with $c_{6}=6727, c_{7}=30160, c_{8}=30765$, $c_{9}=21000, c_{10}=28364, c_{11}=26880, c_{12}=26460, c_{13}=20790$, $c_{14}=10290, c_{15}=8022, c_{16}=2940, c_{17}=4417, c_{18}=2835, c_{19}=210$, $c_{20}=21, c_{21}=1$.

\section{ACKNOWLEDGEMENT}

This research is funded by The Research Center Universitas Lampung under Postgraduate research grant project and the athors thanks for the fund.

\section{REFERENCES}

$\mathrm{Al}$ Etaiwi, W. M. (2014). Encryption algorithm using graph theory. Journal of Scientific Research and Reports, 3(19); 25192527

Álvarez, M. C. and D. Ehnts (2015). The Roads not Taken: Graph Theory and Macroeconomic Regimes in Stock-flow Consistent Modeling. Levy Economics Institute of Bard College, Annandale-on-Hudson

Amanto, A., W. Wamiliana, M. Usman, and R. Permatasari
(2017). Counting The Number of Disconnected Vertex Labelled Graphs with Order Maximal Four. Science International Lahore, 29(6); 1181-1186

Bona, M. (2007). Introduction to Enumerative Combinatorics. McGraw-Hill Science

Brandes, U. and S. Cornelsen (2009). Phylogenetic graph models beyond trees. Discrete Applied Mathematics, 157(10); 2361-2369

Cayley, A. (1874). On the mathematical theory of isomers. The London, Edinburgh, and Dublin Philosophical Magazine and Journal of Science, 47(314); 444-447

Gramatica, R., T. Di Matteo, S. Giorgetti, M. Barbiani, D. Bevec, and T. Aste (2014). Graph theory enables drug repurposing-how a mathematical model can drive the discovery of hidden mechanisms of action. PloS One, 9(1); 84912

Hsu, L. H. and C. K. Lin (2009). Graph Theory and Interconnection Networks. Taylor and Francis Group

Huson, D. H. and D. Bryant (2006). Application of phylogenetic networks in evolutionary studies. Molecular Biology and Evolution, 23(2); 254-267

Kannimuthu, S., D. Bhanu, and K. Bhuvaneshwari (2020). A novel approach for agricultural decision making using graph coloring. SN Applied Sciences, 2(1); 1-6

Kawakura, S. and R. Shibasaki (2018). Grouping Method Using Graph Theory for Agricultural Workers Engaging in Manual Tasks. Journal of Advanced Agricultural Technologies, 
5(3); $173-181$

Mathur, R. and N. Adlakha (2016). A graph theoretic model for prediction of reticulation events and phylogenetic networks for DNA sequences. Egyptian Journal of Basic and Applied Sciences, 3(3); 263-271

Ni, B., R. Qazi, S. U. Rehman, and G. Farid (2021). Some Graph-Based Encryption Schemes. Journal of Mathematics, 2021; 1-8

Pertiwi, F., Amanto, Wamiliana, Asmiati, and Notiragayu (2021). Calculating the Number of vertices Labeled Order Six Disconnected Graphs which Contain Maximum Seven Loops and Even Number of Non-loop Edges Without Parallel Edges. Journal of Physics: Conference Series, 1751(1); 12026

Priyadarsini, P. (2015). A survey on some applications of graph theory in cryptography. Journal of Discrete Mathematical Sciences and Cryptography, 18(3); 209-217

Puri, F., M. Usman, M. Ansori, Y. Antoni, et al. (2021). The Formula to Count The Number of Vertices Labeled Order Six Connected Graphs with Maximum Thirty Edges without Loops. Journal of Physics: Conference Series, 1751(1); 12023

Putri, D., Wamiliana, Fitriani, A. Faisol, and K. Dewi (2021).
Determining the Number of Disconnected Vertices Labeled Graphs of Order Six with the Maximum Number Twenty Parallel Edges and Containing No Loops. Journal of Physics: Conference Series, 1751(1); 12024

Slomenski, W. (1964). Application of the Theory of Graph to Calculations of the Additive Structural Properties of Hydrocarbon. Russian Journal of Physical Chemistry, 38; 700-703

Wamiliana, A. Nuryaman, Amanto, A. Sutrisno, and N. Prayoga (2019). Determining the Number of Connected Vertices Labelled Graph of Order Five with Maximum Number of Parallel Edges is Five and Containing No Loops. Journal of Physics: Conference Series, 1338(1); 12043

Wamiliana, W., A. Amanto, and G. T. Nagari (2016). Counting the Number of Disconnected Labeled Graphs of Order Five without Paralel Edges. Internasional Series on Interdisciplinary Research, 1(1); 4-7

Wamiliana, W., A. Amanto, M. Usman, M. Ansori, and F. C. Puri (2020). Enumerating the Number of Connected Vertices Labeled Graph of Order Six with Maximum Ten Loops and Containing No Parallel Edges. Science and Technology Indonesia, 5(4); 131-135 